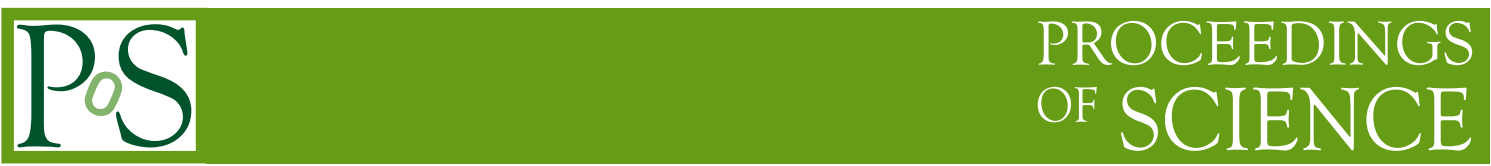

\title{
General relativity experiment with frozen spin rings
}

\author{
András László* \\ Wigner Research Centre for Physics of the Hungarian Academy of Sciences, \\ Konkoly-Thege M. u. 29-33, 1121 Budapest, Hungary \\ E-mail: laszlo.andrasewigner.mta.hu
}

In experimental proposals published in the last two decades, a so called frozen spin storage ring concept was proposed for setting upper experimental bounds to electric dipole moment (EDM) of elementary particles. In a recent paper of ours, a fully covariant general relativistic (GR) calculation was presented on the Earth's gravitational modification effect in such mixed magnetic-electric frozen spin storage ring on the spin transport, which could contribute to such measurement. It was shown that similarly to an EDM signal, GR causes a spin precession out of the orbital plane, under the frozen spin condition. The rate of the vertical polarization buildup is predicted to be $-a \beta \gamma \mathbf{g} / c$, where $\mathbf{g}$ is the gravitational acceleration on the surface of the Earth, $c$ is the speed of light, $\beta \gamma$ is the particle momentum over mass, and $a$ is its magnetic moment anomaly. It is seen that the effect increases unboundedly with the Lorentz factor $\gamma$. Moreover, it is proportional to the magnetic moment anomaly $a$. This paper mainly addresses the experimental perspectives to detect this effect in a realistic frozen spin storage ring configuration. Such a measurement would provide a novel test of GR, sampling the tensorial nature of GR at a microscopic level, as acting on the spin vector of elementary particles. The conclusion is that the pertinent GR experiment seems to be realistic with large magnetic moment anomaly particles, such as tritons, helion3 or protons, whereas it is not realistic with small magnetic moment anomaly particles, such as deuterons, muons or electrons.

23rd International Spin Physics Symposium - SPIN2018 -

10-14 September, 2018

Ferrara, Italy

${ }^{*}$ Speaker. 


\section{Introduction}

In a recent set of papers [1, 2, 3] a claim was made by Morishima et al that, in the muon anomalous magnetic moment experiments, also called g-2 experiments [ [ 6 , 6, 6, 元], there can be an unaccounted systematic error caused by the gravitational field of the Earth through its general relativistic (GR) action on spin vectors. Various GR authors [8, 9] responded with heuristic estimates, claiming that the effect must be way smaller. Further GR authors [10] argued that the effect exactly cancels. Also, due to the non-geodesic nature of the beam orbit, the usual formulae of de Sitter and Lense-Thirring precession [11] (also called geodetic effect) do not apply as they are. Motivated by the above controversy, a fully covariant GR calculation was performed by us in [12] for quantifying this effect in the g-2 rings. It was shown that the final experimental systematics caused by GR is nonzero, but is indeed way too small to disturb the measurement. This smallness of the GR systematics is, however, a result of a not entirely trivial effect. First of all, the GR contribution is ab initio small in comparison to the spin precession rate due to magnetic field in a $\mathrm{g}-2$ ring setting. Secondly, it turns out that the GR systematics comes as a beam-radial precession vector, which adds vectorially to the otherwise vertical magnetic precession vector caused by $\mathrm{g}-2$, thus making the GR modification in the observed precession frequency to be only of second order. In conclusion, the GR systematics in $\mathrm{g}-2$ rings caused by the gravitational field of the Earth is negligible.

As pointed out, the absence of substantial GR contribution by Earth's gravitational field to g-2 experiments is partly a result of a second order suppression, caused by the geometry of the particular ring configuration. The pertinent GR modification effect, however, can be brought up to first order if the guiding fields of the particle beam is configured such that the spin vector in the comoving frame does not feel any torque by the guiding fields. Such a configuration is called a frozen spin storage ring [13, 14, 15], and that concept was mainly invented for experimental determination of electric dipole moment (EDM) [15] of charged particles with spin. Like since a decade ago, it was realized by other authors that in a frozen spin ring the Earth's gravity is likely to have a systematic contribution [16, 17, 18, 19, 20]. These approximative calculations captured well the qualitative behavior of the GR contribution, and indicated that if the design sensitivity of EDM rings intends to reach $\approx 10^{-29} \mathrm{ecm}$, the GR effect needs to be considered. In order to quantitatively capture the magnitude of the effect accurately, a fully covariant GR calculation was performed by us in [12]. The calculation shows that in a frozen spin configuration, Earth's gravitational field causes an out of the orbital plane precession at a rate

$$
-a \beta \gamma \underbrace{\mathbf{g} / c}_{\approx 33 \mathrm{nrad} / \mathrm{sec}}
$$

where $\mathbf{g}$ denotes the gravitational acceleration at the surface of the Earth, $c$ is the speed of light, $a$ is the magnetic moment anomaly of the particle, and $\beta \gamma$ is the momentum over mass of the particle. It is seen that the effect is proportional to the magnetic moment anomaly $a$, and that it grows unboundedly with the Lorentz factor $\gamma$. Since the effect is in principle unbounded, the question naturally arises: can one specifically design an experiment such that this GR contribution is well measurable? One could think, for instance, about optimizing EDM rings such that this 
effect becomes dominant. If such an experiment could be carried out, it would test GR in a very interesting condition:

- with microscopic particles,

- at relativistic speeds,

- along non-geodesic (forced) trajectories,

- tensorial nature of GR would be at test and not merely the gravitational drag.

Such a configuration would be a microscopic analogy of the Gravity Probe B satellite experiment [21. 22], where GR modification of free gyroscopic motion around Earth was measured. However, in contrast to satellite experiments, in frozen spin rings the general relativistic gyroscopic motion would be tested along non-geodesic (forced) trajectories. That is, the GR correction to the Thomas precession could be experimentally tested.

\section{Relativistic motion of charged point particle with spin, in GR}

In a special relativistic or general relativistic framework, the motion of a point particle, i.e. of a particle with irresolvable internal structure is encoded via a future directed timelike worldline in spacetime. At each point of its worldline, it has a corresponding tangent vector, which can be normalized to unit length, and is then called the four velocity $u^{a}$. Moreover, if the particle has a spin, then at each point of the worldline, a unit length spacelike vector $w^{a}$ describes the instantaneous spin direction, being always orthogonal to the four velocity $u^{a}$ due to its quantum mechanical origin. This is illustrated in Figure 1 .

$u^{a}$ denotes the four velocity of the particle at points of the trajectory

$w^{a}$ denotes the spin direction four vector at points of the trajectory

(one has the orthogonality constraint $g_{a b} u^{a} w^{b}=0$ at each point)

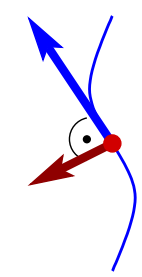

Figure 1: Illustration of the spacetime evolution of a point particle with spin. The particle evolves along some future directed worldline with instantaneous four velocity $u^{a}$. At the points of the worldline a spin direction vector $w^{a}$ is also present. At each point of the worldline one has the constraints $g_{a b} u^{a} u^{b}=1$, $g_{a b} w^{a} w^{b}=-1$ and $g_{a b} u^{a} w^{b}=0$ where $g_{a b}$ is the spacetime metric tensor field with signature (+,-,-,-).

The evolution of the trajectory is governed by the relativistic Newton equation, and along the worldline the evolution of the spin direction vector is governed by the Thomas-Bargmann-MichelTelegdi (TBMT) equations [23, 24, 25, 26]:

$$
\begin{aligned}
& \text { (A) } u^{a} \nabla_{a} u^{b}=-\frac{q}{m} g^{a b} F_{a c} u^{c}, \quad(\leftarrow \text { relativistic Newton eq. }) \\
& \text { (B) } \quad D_{u}^{F} w^{b}=-\frac{\mu}{s}\left(g^{a b} F_{a c}-u^{b} u^{d} F_{d c}-g^{a b} F_{a d} u^{d} u^{e} g_{e c}\right) w^{c}, \quad(\leftarrow \text { TBMT eq. }) \\
& +\frac{d}{s}\left(g^{a b \star} F_{a c}-u^{b} u^{d \star} F_{d c}-g^{a b \star} F_{a d} u^{d} u^{e} g_{e c}\right) w^{c} \text {. }
\end{aligned}
$$


In the above equations $m, q, s$ denote particle mass, charge and spin magnitude $\left(s=\frac{1}{2}, 1, \frac{3}{2}, \ldots\right)$, whereas $\mu$ and $d$ denote the magnetic and electric dipole moment of the particle, respectively. The electric dipole moment $d$ is usually extremely small, and the aim of the EDM experiments is to obtain an upper bound to the quantity $d$. The field quantities in the above equations are as follows: $F_{b c}$ denotes the electromagnetic field strength tensor of the guiding fields, ${ }^{\star} F_{b c}$ denotes the Hodge dual of the electromagnetic field strength tensor, $g_{a b}$ denotes the spacetime metric tensor field as usual, $\nabla_{a}$ denotes the spacetime covariant derivation compatible with the metric, and $D_{u}^{F} w^{b}$ denotes the Fermi-Walker derivative of $w^{b}$ along the worldline described by $u^{a}$, defined as $D_{u}^{F} w^{b}=u^{a} \nabla_{a} w^{b}+g_{e d} w^{e} u^{b} u^{a} \nabla_{a} u^{d}-g_{c d} w^{c} u^{d} u^{a} \nabla_{a} u^{b}$. As it is well-known, the geometric meaning of the covariant derivative of vectors is the infinitesimal parallel transport, whereas the meaning of Fermi-Walker derivative is the infinitesimal parallel transport of rigid orthonormal frames (preserving orthonormality). As such, the spin evolution equation for free gyroscopes would be $D_{u}^{F} w^{b}=0$, and the Gravity Probe B satellite experiment [21, 22] tested this kinematic equation. Whenever one has geodesic motion $\left(u^{a} \nabla_{a} u^{b}=0\right)$, the kinematic effect of the gyroscopic motion is called geodetic effect (de Sitter or Lense-Thirring precession). Along a forced trajectory ( $u^{a} \nabla_{a} u^{b}=$ some four force), the kinematic effect of the gyroscopic motion is called Thomas precession, which is the case for particles in Earth-based storage rings. Moreover, the explicit torque exerted on the spin by the electromagnetic field, i.e. by the right hand side of Eq.(2.1)(B), is called Larmor precession. The Thomas and Larmor precession is already present in the special relativistic (flat spacetime) case. The frozen spin condition means that assuming the absence of gravity and of EDM, one tries to construct a configuration in which the Thomas+Larmor precession vanishes.

\section{Frozen spin (EDM) rings and GR modification of spin dynamics within}

The main idea of frozen spin storage rings [13, 14, 15] is that in an idealized case one injects longitudinally spin polarized particle beams into a planar circular storage ring, where the magnetic bending field $(B)$ is homogeneous vertical, and there is a horizontal cylindric, i.e. beam-radial electric bending field $(E)$ in addition. Given the particle type, the bending fields $B$ and $E$ are configured such that both the relativistic Newton equation Eq.2.1)(A) is satisfied, moreover the particle spin, which is governed by the TBMT equation Eq.(2.1)(B), does not precess against the beam-tangential direction in the horizontal plane (horizontal frozen spin condition). If no EDM of the particle and no gravitational fields are present, then the spin would not precess at all in such a configuration against the beam-tangential direction (frozen spin condition). Whenever an EDM of the particle would be present, the spin would precess out of the orbital plane under this condition. Similarly, the gravitational field of the Earth through GR causes an out of the orbital plane precession, which was qualitatively determined in [16, 17, 18, 19], and quantitatively determined in [12, 20]. Given the particle mass $m$, charge $q$, spin $s$, gyromagnetic factor $\mathrm{g}=\frac{2 m \mu}{q s}$, magnetic moment anomaly $a=\frac{\mathrm{g}-2}{2}$, as well as its momentum over mass $\beta \gamma$, and ring radius $r$, the Newton equation for the circular motion and the frozen spin condition are simultaneously satisfied whenever

$$
\begin{aligned}
E \cdot r & =-\operatorname{sign}(a) \frac{m c^{2}}{q} \frac{(a \beta \gamma)^{2} \sqrt{a^{2}+(a \beta \gamma)^{2}}}{a^{2}(1+a)}, \\
B \cdot r & =\frac{m c}{q} \frac{(a \beta \gamma)\left(a-(a \beta \gamma)^{2}\right)}{a^{2}(1+a)}
\end{aligned}
$$


holds (two equations for the two unknowns $B$ and $E$ ). Under this condition, assuming negligible EDM and GR effects, the longitudinal spin polarization of the beam is preserved, i.e. no vertical polarization buildup shall be present. If a small nonvanishing particle EDM is present, that would cause a slow vertical spin polarization buildup, which the EDM experiments would aim to detect. On the other hand, assuming negligible EDM, but non-negligible GR effect by Earth's gravitational field, the following ingredients are affected:

- The metric and thus the parallel transport $\nabla_{a}$ and Fermi-Walker transport $D^{F}$ are modified. (The Newton + TBMT equations Eq.(2.1) are affected by GR, quite naturally.)

- The Maxwell equations $g^{a b} \nabla_{a} F_{b c}=0, g^{a b} \nabla_{a}{ }^{\star} F_{b c}=0$ are modified.

(The electromagnetic fields of the storage ring are affected by GR, as illustrated in Figure \&)
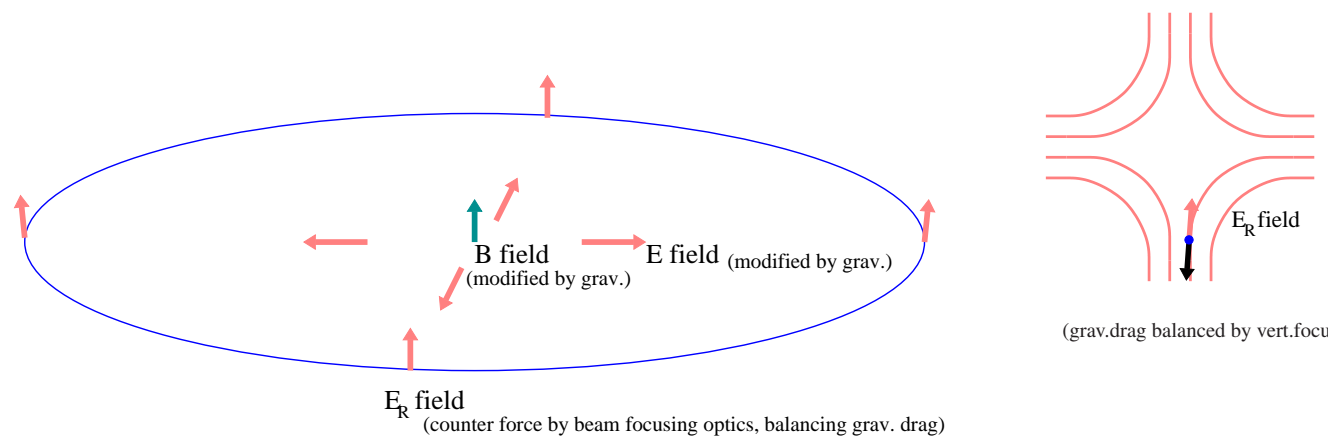

(grav.drag balanced by vert.focusing)

Figure 2: Illustration of the electromagnetic fields in a frozen spin storage ring, with combined magnetostatic and electrostatic bending fields $(B$ and $E$ ), and with electrostatic vertical focusing beam optics. The geometry of the bending fields need to be defined over the curved spacetime due to the gravitational field of the Earth. Moreover, Earth's gravitational drag is equilibrated by the Earth-radial electrostatic field $\left(E_{R}\right)$, exerted by the electrostatic vertical beam focusing optics at the place of the nominal equilibrium beamline.

In [12] it is shown that the total GR effect induces an out of the orbital plane precession at a rate Eq. (1.1) in a generic mixed magnetic-electric frozen spin ring with electric vertical focusing. An earlier calculation [20] quantified the same thing for the special case of electrostatic-only frozen spin rings (only possible for $a>0$ and at magic momentum $|\beta \gamma|=\frac{1}{\sqrt{a}}$ ). It can be shown that the GR prediction differs from the prediction of semi-classical treatment of gravity at a ratio $a:(1+a)$, and thus the experimental verification of the effect would be truly a GR test. (Semi-classical treatment: one prescribes by hand the four-force of the gravitational drag on the particle as coming from GR, i.e. as coming from the gravitational acceleration, but one does the calculation over flat spacetime.)

\section{Optimalization}

From Eq.(1.1) it is seen that one can increase the GR effect via trying to reach as large $|a \beta \gamma|$ as possible. However, from Eq.(3.1) it is seen that for a given particle type (with fixed $\frac{m}{q}$ and $a$ ), the necessary electric bending power $|E \cdot r|$ for maintaining the frozen spin condition increases rapidly, as $\sim O\left(|a \beta \gamma|^{3}\right)$. Since too large ring radius $r$ or too large electric fields $|E|$ are unrealistic, this sets limits to the experimental possibilities. One can realize, however, that because of Eq. (3.1), for a fixed $|a \beta \gamma|$, the necessary electric bending power $|E \cdot r|$ decreases as $\sim O\left(|a|^{-2}\right)$. Thus, it is 
advantageous to use particles with large magnetic moment anomaly $|a|$. Using particle data table of [27], a set of particle species can be proposed for conducting such a frozen spin GR experiment. Assume that a reasonable GR signal strength of $|a \beta \gamma|=0.4$ is aimed to be achieved, which would result in an out of the orbital plane precession (vertical polarization buildup) due to GR at a rate $13.1 \mathrm{nrad} / \mathrm{sec}$, according to Eq. 1.1. Assume that the main bottleneck of feasibility, namely the electric field strength, is also fixed to a reasonable value $|E|=4.10 \mathrm{MV} / \mathrm{m}$. Then, feasible and unfeasible configurations are listed in Table 1 .

Particle species with which frozen spin GR experiments may be conducted:

\begin{tabular}{c|c|c|c|c|c|c} 
particle name & $|a \beta \gamma|$ & $r[\mathrm{~m}]$ & $|E|[\mathrm{MV} / \mathrm{m}]$ & $|B|[$ Tesla $]$ & $p[\mathrm{MeV} / \mathrm{c}]$ & $\mathscr{E}_{\mathrm{kin}}[\mathrm{MeV}]$ \\
\hline triton & 0.4 & 1.55 & 4.10 & 0.0335 & 141.9 & 3.58 \\
helion3 & 0.4 & 4.13 & 4.10 & 0.0353 & 268.5 & 12.8 \\
proton & 0.4 & 7.50 & 4.10 & 0.0304 & 209.7 & 23.1
\end{tabular}

Particle species with which frozen spin GR experiments are unrealisticly expensive:

\begin{tabular}{c|c|c|c|c|c|c} 
particle name & $|a \beta \gamma|$ & $r[\mathrm{~m}]$ & $|E|[\mathrm{MV} / \mathrm{m}]$ & $|B|[\mathrm{Tesla}]$ & $p[\mathrm{MeV} / \mathrm{c}]$ & $\mathscr{E}_{\mathrm{kin}}[\mathrm{MeV}]$ \\
\hline deuteron & 0.4 & 1796 & 4.10 & 0.0243 & 5283 & 3731 \\
electron & 0.4 & 5942 & 4.10 & 0.0136 & 176.5 & 176.0 \\
muon & 0.4 & 1228520 & 4.10 & 0.0136 & 36497 & 36391
\end{tabular}

Table 1: Top table: list of particle species with which realistic frozen spin GR experiments can be conducted. Some realistic example configurations are also listed along with the particle types. Namely, the predicted GR signal strength is fixed to $|a \beta \gamma|=0.4$, meaning an expected vertical polarization buildup rate of $13.1 \mathrm{nrad} / \mathrm{sec}$. At the same time the electric bending field, being the main bottleneck, is fixed to a technically realistic value $|E|=4.10 \mathrm{MV} / \mathrm{m}$. Bottom table: list of particle species with which frozen spin GR experiments are unrealistically expensive. Those particles all have small magnetic moment anomaly $|a|$.

The biggest systematic errors in such an experimental setting is expected to come from the conical imperfections of the magnetic bending field. That is, besides the vertical component $B$, there can be a small beam-radial magnetic field component $B_{r}$. Assuming a planar circular beam orbit at equilibrium on the Earth's surface satisfying the horizontal frozen spin condition, then its vertical polarization buildup rate is predicted to be

$$
\underbrace{-\frac{q(1+a)}{m} \frac{1}{\gamma^{2}} B_{r}}_{\text {magnetic field conicality imperfection term }}+\underbrace{-a \beta \gamma \mathbf{g} / c}_{\text {GR term }}
$$

due to magnetic field conical imperfection and GR. The magnetic field conical imperfection $\frac{B_{r}}{B}$ is normally rather hard to suppress beyond certain limits. In order to overcome that limitation, in [28] a new concept was proposed: two different particle species are proposed to be studied in the same frozen spin storage ring, called to be a doubly-frozen spin ring. That turns out to be kinematically possible for certain pairs of particle species, at unique beam momenta and bending field configurations. Given such a setting, one has two vertical polarization buildup observables Eq.(4.1) for the two particle beams, and one can use these to create an optimal linear combination 
of these observables which is insensitive to $B_{r}$ to the first order. In that way, the extreme sensitivity to the magnetic field imperfections can be suppressed.

\section{Concluding remarks}

In this contribution a general relativity (GR) experiment was proposed in order to measure the effect of Earth's gravitational field on the spin transport in frozen spin particle storage rings. Since electric dipole moment (EDM) experiments are already planned to be performed using frozen spin rings [13, 14, 15], it is quite natural to try to measure this effect as part of their program. GR causes a precession out of the orbital plane at a rate $-a \beta \gamma \mathbf{g} / c$ [12], where $\mathbf{g}$ is the gravitational acceleration at the surface of the Earth, $c$ is speed of light, $a$ is the magnetic moment anomaly of the particle, and $\beta \gamma$ is the momentum over mass of the particle. The constant $\mathrm{g} / c$ is about $\approx 33 \mathrm{nrad} / \mathrm{sec}$. Since the effect is proportional to the product $a \beta \gamma$, it is advantageous to have as large $|a \beta \gamma|$ as possible. The issue is caused by the fact that the necessary electric bending field increases very fast, as $\sim O\left(|a \beta \gamma|^{3}\right)$ for a fixed particle type. However, at a fixed anticipated GR signal strength, the necessary electric bending field decreases as $\sim O\left(|a|^{-2}\right)$, thus it is advantageous to use particles with large $|a|$. A relatively large GR signal strength $\approx 10 \mathrm{nrad} / \mathrm{sec}$ can be met with a realistic bending radius $\approx 10 \mathrm{~m}$ and a realistic electric bending field $\approx 5 \mathrm{MV} / \mathrm{m}$ with moderate energy triton, helion 3 or proton beams, due to their large magnetic moment anomaly $|a|$. Particles with small $|a|$ such as deuterons, muons or electrons are not realistic options. The main systematics, caused by magnetic bending field imperfections can be substantially reduced via the method of doubly-frozen spin ring concept [28] when in the same bending fields two beams with different particle species are compared. This opens up an experimental possibility to test GR in a very interesting regime: with microscopic particles, at relativistic speeds, along non-geodesic (forced) trajectories, and to test the tensorial nature of GR under these conditions - not merely the gravitational drag. The pertinent GR signal is distinguishable from a possible EDM signal via their opposite space reflection properties, i.e. via their opposite sign change when reverting beam direction.

\section{Acknowledgments}

This work was supported in part by the Hungarian Scientific Research fund (NKFIH 123842123959).

\section{References}

[1] T. Morishima, T. Futamase: Post-Newtonian effects of Dirac particle in curved spacetime - I : magnetic moment in curved spacetime; [arXiv:1801.10244].

[2] T. Morishima, T. Futamase, H. M. Shimizu: Post-Newtonian effects of Dirac particle in curved spacetime - II : the electron g-2 in the Earth's gravity; [arXiv: 1801.10245$].$

[3] T. Morishima, T. Futamase, H. M. Shimizu: Post-Newtonian effects of Dirac particle in curved spacetime - III : the muon g-2 in the Earth's gravity; [arXiv:1801.10246].

[4] The Muon g-2 experiment [http://muon-g-2. fnal.gov]. 
[5] G. W. Bennett et al: Phys. Rev. D 73 (2006) 072003.

[6] J. P. Miller, E. de Rafael, B. L. Roberts: Rept. Prog. Phys. 70 (2007) 795.

[7] S. R. Mane, Y. M. Shatunov, K. Yokoya: Rept. Prog. Phys. 68 (2005) 1997.

[8] M. Visser: Post-Newtonian particle physics in curved spacetime; [arXiv: 1802.00651 ].

[9] P. Guzowski: The effect of Earth's gravitational field on the muon magic momentum; [arXiv:1802.01120].

[10] H. Nikolic: Can effective muon g-2 depend on the gravitational potential?; [arXiv:1802.04025].

[11] C. Lämmerzahl, G. Neugebauer: The Lense-Thirring Effect: From the Basic Notions to the Observed Effects; Lecture Notes in Physics, vol 562 Springer (2001).

[12] A. László, Z. Zimborás: Class. Quant. Grav. 35 (2018) 175003.

[13] Y. Senichev et al: Proceedings of IPAC2017 (2017) TUPVA084, 2275.

[14] Y. Semertzidis: Eur. Phys. J. Web. Conf. 118 (2016) 01032.

[15] R. Talman: The Electric Dipole Moment Challenge; IOP Concise Physics (2017).

[16] A. Kobach: Nucl. Phys. B911 (2016) 206.

[17] A. J. Silenko, O. V. Terayev: Phys. Rev. D76 (2007) 061101.

[18] Y. N. Obukov, A. J. Silenko, O. V. Terayev: Phys. Rev. D94 (2016) 044019.

[19] Y. N. Obukov, A. J. Silenko, O. V. Terayev: Int. J. Mod. Phys. A31 (2016) 1645030.

[20] Y. Orlov, E. Flanagan, Y. Semertzidis: Phys. Lett. A376 (2012) 2822.

[21] Gravity Probe B experiment [https://einstein.stanford.edu].

[22] C. W. F. Everitt et al: Phys. Rev. Lett. 106 (2011) 221101.

[23] M. Conte, R. Jagannathan, S. A. Khan, M. Pusterla: Particle Accelerators 56 (1996) 99.

[24] J. D. Jackson: Classical Electrodynamics; Wiley (1999).

[25] S. Hawking, G. F. R. Ellis: Large Scale Structure of Spacetime; Cambridge University Press (1973).

[26] R. M. Wald: General Relativity; Chicago University Press (1984).

[27] N. J. Stone: Atomic Data and Nuclear Data Tables 90 (2005) 75.

[28] R. Talman: A doubly-magic storage ring EDM measurement method; [arXiv: 1812.05949 ]. 